\title{
Predict Link Failure in AODV Protocol to provide Quality of Service in MANET
}

\author{
Sedrati Maamar \\ LaSTIC, Computer Science Department, Batna 2 University, Algéria \\ E-mail: msedrati@gmail.com \\ Benyahia Abderezzak \\ LaSTIC, Computer Science Department, Batna 2 University, Algéria \\ E-mail: benyahia.abderrezak@yahoo.fr
}

\begin{abstract}
Quality of Service (QoS) in Ad hoc networks (MANETs) and more precisely in routing is the subject of several studies with the aim of providing better solutions for new applications requiring high throughput and very low delay.

The objective of this work is to enhance the AODV (Ad hoc On Demand Distance Vector) routing strategy in maintenance phase, to improve QoS. It aims to add a mechanism able to predict link failure in use based on signal strength which is able to determine if the quality of link will be improved (i.e. stable) or more bad (i.e. probability of failure) in order to allow us not only to make the link-management more robust but to anticipate on link breaking and improve QoS. When signal quality is declining due to neighbor node remoteness, a discovery of a part of road rescue with two hops will be established and it will be used when disconnection happens. Simulations under Network Simulator (Ns2) were conducted to measure traffic control, packets delivery and lost ratio in original protocol and modified version which are presented in this paper.
\end{abstract}

Index Terms - Mobile Ad hoc Networks (MANET), AODV protocol, QoS, routing, signal strength, stable connection, Network Simulator NS2.

\section{INTRODUCTION}

Currently, mobile Ad hoc networks (MANETs) should be able to provide a quality of service for new needs of multimedia applications and real time that require high throughput and reduced delays, so it is imperative that networks can be able to provide quality of service (QoS) in terms of throughput, delay, jitter, reliability, etc. taking into account limitations imposed by such networks like shared medium and dynamic topology.

RFC 2386 [1] characterizes QoS as a series of needs to be provided by network to transport traffic from source to destination. It is defined in [2] such as the ability of network component (router, node, etc...) to provide security level for transmitting data. It is very important in Ad hoc networks because it can improve performance and enable flow of data [3]

QoS must be assured at different levels in network architecture. Ad hoc network is a wireless network, without infrastructure in which resources (bandwidth, energy, etc...) are limited. Routing in these networks is the interest's heart of researchers of researchers. Several solutions have been explored and many protocols have been developed [4] including AODV.

Nevertheless, initial versions of Ad hoc routing protocols doesn't take into account real-time constraints to support applications that require certain QoS. So they must be adapted appropriately to meet QoS requirements of these new applications. A QoS routing protocol select roads that best meets QoS parameters like throughput, delay, jitter, etc... to better meet specific requirements of applications.

In this paper, we propose to reinforce AODV routing strategy especially in the maintenance phase.

For this, we aim to add mechanism able to predict link failure in use, based on signal strength that determines if link quality improves (more stable) or decreases failure probability which allows us not only to make link management more robust, and anticipate the link failure and therefore to optimize some QoS parameters and to determine impact of mobility and duration interval between two consecutive rate on traffic control, reliability (packet loss), packets transit delay etc...

The rest of this paper is organized as follows. Section 2 introduces some related works. Section 3, deals with a description of QoS routing and different models cited in literature. AODV protocol, discovery and maintenance phases will be the subject of section 4. In Section 5, we present PF_AODV (Predict Failure in AODV) the proposed protocol. Section 6 is devoted to simulation model description used under network simulator (Ns2) and results interpretation and comparison between AODV and PF_AODV then we will finish with conclusion and feather recommendations of our research.

\section{RELATED WORK}

In recent years, several solutions have been proposed to support QoS at routing level for mobile ad hoc networks (MANETs). Most of them extend AODV protocol [5] and use either backup routes selected by multi paths protocols that generate a significant overhead 
during discovery and maintenance phases, or a link failure prediction mechanism based on residual energy or received signal value from neighbor node, or a combination of both of them (prediction \& multi-path).

Authors in [6 - 8, 10 - 13] use signal strength to predict path stability or link failure to evaluate route stability and reliability, route life time in multi-path context with AODV protocol. Wang and Lee [6] propose a reliable multi-path QoS routing (RMQR) protocol with a slot assignment scheme. This protocol uses route life time and hops number to select a path with low latency and high stability. Global positioning system (GPS) is used to determine route expiration time between two connected nodes. RMQR protocol has some outstanding properties compared to others protocols.

Cha et al [7] propose an efficient routing mechanism with route prediction of nodes for link reliability in MANETs. Each node with GPS predicts to move expected location by calculating node's location and velocity which enables source node to choose the route with longest connection time among multiple routes. This solution reduces unnecessary control messages in MANETs.

AODV - Reliable Delivery (AODV-RD) developed in [8] focuses on an anticipated warning mechanism to detect a link failure by Signal Stability-Based Adaptive Routing (SSA) [9], method to identify good or bad link based on weakness or greatness of signals and to proceed a repair action before the primary route breaks. This protocol increase PDR and reduce end to end delay.

Abdule and Hassan [10] propose Divert Failure Route Protocol (DFRP) for resolving link failure problem in Ad hoc network based on AODV protocol. DFRP tries to avoid a link failure in advance and monitors the link to next hop to predict link status through signal strength. Main functions of DERP are to predict signal strength, and to find a new route to divert data to the new path. This protocol reduces delay resulting from sending link failure information back to the sender.

Hwang and Varshney [11] propose an Adaptive Dispersity QoS Routing (ADQR) method to find multiple disjoint routes with long lifetime. ADQR use signal strength information obtained from lower layers to predict route failure and initiate fast route maintenance to react quickly to network changes. It improves routing performance and supports end-to-end QoS.

AODV-RSS (AODV with received signal strength) a long-lived path for ad hoc networks cited in [12] uses the received signal strength (RSS) and received signal strength changing rate to predict the link available time (LAT) and to find a route that can sustain longer. This protocol can greatly improve quality of routing path, and route reestablishment frequency.

Sarma and Nandi [13] develops a Route Stability based QoS Routing (RSQR) protocol in MANETs to support QoS routing requirements on throughput and delay. This protocol computes link stability and route stability based on received signal strengths. The route stability information can be utilized to select a route with higher stability among all feasible routes. RSQR show performance improvements in terms of packet delivery ratio, control overhead and average end-to-end delay.

Works cited in [14 - 17] are based only on route stability and reliability combined to multi paths and/or AODV protocol. Boshoff and Helberg [14] extend AODV routing protocol to support multi-path detection for MANETs. End-to-end delay is used as metric for route selection instead of hop count. When route failure occurs, alternate route is used. This protocol shows significant improvements in delay, packet delivery fraction and routing overhead compared to others protocols.

Ghanbarzadeh and Meybodi [15] optimize AODV routing protocol performance by using link availability prediction in urban area by Hello message mechanism. This strategy reduces message overhead and average of broken links metrics relative to classic AODV.

Modified Reverse Ad Hoc On-demand Vector (MRAODV) routing protocol cited in [16] is based on link/route stability estimation which reduces routing overhead in discovery and maintenance process and increased packet delivery ratio. When an active route fails, the source node with the awareness of routes stabilities, can select the best path in available routes set.

A proposal discussed in [17] is an intelligent protocol that performs proactive route maintenance process by using path reliability information, and analyzes its effect in path selection. When route failure occurs, packets may be rapidly switched to an alternate route, without waiting until the route to be broken to restart the route discovery process. This protocol outperforms AODV in terms of increased throughput and reduced overhead.

The most of these proposals [18 - 22] improve AODV protocol and use Failure Prediction mechanism, network lifetime and/or energy consumption. Maleki, Mortez et al [18] propose Lifetime Prediction Routing (LPR) to enhance the network lifetime by finding routing solutions that minimize the variance of the remaining energies of nodes in the MANET. They use battery lifetime prediction based on its past activity and they choose the path with maximum lifetime. LPR introduces some additional traffic but it improves the network lifetime.

Veerayya [19] develops a Stability-based QoS-capable AODV (SQ-AODV) protocol to enhance AODV based on how to best use node energy for both route discovery and maintenance process and how to adapt more quickly to networks conditions. SQ-AODV provides stable routes, increase PDR, and reduce control overhead and packet delay.

A proposal discussed in [20] called AODV_LFF routing protocol introduces the mechanism of link failure prediction into AODV routing protocol in data transmission process. This protocol can reduce network transmission delay effectively, and also can boost packet delivery rate.

Haghighat and Khoshrodi [21] propose a Stable QoS based on AODV protocol. Their fundamental idea is to separate nodes and links that can be used in a QoS routing and try to create more stable path to support QoS. This approach can reduce route breaking meanwhile 
improving packet delivery.

Satyanarayana and Rao [22] proposed Link Failure Prediction QoS Routing (LFPQR) protocol for MANETs. LFPQR predicts the node future state to decide whether the node will be selected as a good router or not. The future prediction heuristic depends on mobility and node power level. The most stable paths must be selected and therefore QoS requirements could be satisfied. In LFPQR packet loss and end-to-end packet delay are reduced and it outperforms in high mobility conditions and/or at frequent node failures.

\section{QUALITY OF SERVICE IN MANET}

Quality of Service (QoS) refers to capacity to provide better service for different applications having ability to transmit under good conditions data stream, in terms of availability, throughput, delay, jitter, packet loss... Quality of service also involves controlling and optimizing network resources.

QoS model in mobile ad hoc network defines architecture for all possible. This template must take into account all these networks constraints (mobility, energy lack, etc...) [3, 23, 24].

Classical models like Intserv [25] and DiffServ [26] proposed first for wired network and Internet are not adapted to MANETs constraints like mobility and capacity.

Various specific models developed for MANETs like FQMM [27] (Flexible Quality of Service Model for Manet) which was the first proposed model with size not exceeding 50 nodes. It doesn't take into account all MANETs characteristics. SWAN model [28] (Service Differentiation in Stateless Wireless Ad Hoc Networks) based on best-effort service and use control admission to check bandwidth availability to ensure traffic transit without congestion. IMAQ [29] (an Integrated Mobile Ad hoc QoS framework) is QoS model oriented for multimedia; it includes a routing layer and software service layer (middleware).

On other hand, QoS routing in MANETs is an essential element and many research try to find a solution to this. Since routing in network, can establish the shortest link in terms of hops or delay between source and destination, the purpose of QoS routing is to find best road according to specific criteria for desired QoS (delay, loss rate, throughput ...) based on reliable and stable links [30, 31].

Several solutions of MANETs routing protocols [32] have been proposed and among them: Ticket-Based QoS Routing [33] designed for networks with low mobility and traffic is managed by tickets allocation. CEDAR [34] (Core-Extraction Distributed Ad hoc Routing algorithm) designed for cluster-based Ad hoc networks in order to reduce control overhead. INSIGNIA protocol [35] adapted to Ad hoc networks, it combines QoS layers notion (base and enhancement layers) by incorporating it's signaling into data packet headers.

\section{AODV PROTOCOL}

\section{A. Introduction}

AODV protocol (Ad-hoc on demand Distance Vector) $[5,36]$ is a reactive routing protocol based on the distance vector algorithm where path between two nodes is calculated when needed (i.e. when a node wants to send data packets it initiates Discovery Phase to find a new path, uses it during the transfer phase, and it must maintain it during utilization (Maintenance Phase).

A set of control packets such RREQ (Route REQueset), RREP (Route Reply), RERR (Route Error), RRepAck (Route Reply Acknowledgment) and Hello messages (Hello) are used in discovery and maintenance process.

In AODV protocol, a routing table is associated for each node to store information as Dest address, active neighbors list, hops number to reach the destination, TTL after which an entry in the table becomes invalid, and so on.

The use of sequence numbers eliminates the formation of infinite loop, limiting control packets transmission (i.e. overhead phenomena) and allows the use of fresh paths in node mobility case, as they help to ensure routing information consistency and coherence [5, 24]

\section{B. Discovery Phase}

Every time, a node wishes to transmit, it checks its routing table for any valid road to desired destination. If isn't the case, it lunches the discovery phase. This operation is initiated by broadcasting RREQ control packet specifying parameters such as sequence number to be used to indicate fresh roads, pair (ID packet and IP source address) to check if request is already treated by node or not (problems of duplication) and TTL (Time To Live) (number of hops) that is assigned to the initial value TTL_START [36].

If no response is made after RREP_WAIT_TIMEOUT period, the same RREQ is rebroadcast by source node but with a TTL incremented by TTL_INCREMENT (more hops, and therefore more chance to find a road) and waiting period for response has longer time than the previous one $[24,36]$.

When an intermediate node receives a request (RREQ), it checks its routing table for availability of path to the destination and if so, a reply packet (RREP) is returned to source telling him how to reach the destination. Otherwise it increments hop count and rebroadcasts RREQ packet. Before sending, node stores source IPs and node from which a first copy of application is received, he will use it to construct reverse path to be traversed by the RREP packet in unicast [24].

When the RREQ packet reaches the destination node, the latter constructs a RREP packet and forwards it in reverse, using previously saved IPs and at each passage by node in reverse path, field "hop count" of this packet (RREP) is incremented (distance in number of hops) [24]. 


\section{Maintenance Phase}

In order to maintain consistent roads, periodic transmission of HELLO message (which is a RREP with TTL equal to one) is performed. If three HELLO messages are not received consecutively from neighboring node, the link in question is considered as failing. Paths failures are generally due to nodes mobility in Ad hoc Network. If unsuccessful, the source node tries to find another path and decrements the attempts number (RREQ_RETRIES) by one [24].

\section{PF_AODV (PREDICT FAILURE IN AODV)}

\section{A. Introduction}

In this section, we start by a motivation with an illustrative example to better promote this solution. Approach principle is based on a discovery phase identical to AODV and a maintenance phase where new algorithms are integrated to predict link failure before a disconnection takes place.

\section{B. Motivations}

In dynamic environment, a frequent disconnection cause's considerable packets data loss due to lack of alternate paths (routes) and the reconstruction of new route generate an additional volume packets (routing) control [37]. That's why it is important to predict any disconnection (failure) probable on active route based on signal strength between neighboring nodes. The signal strength value informs us about the link quality and it depends on nodes mobility. Signal strength can determine if link quality improves (more stable) or decreases (probability of failure) which allow us not only to make link management more robust, and anticipate on link failure and thereby improve QoS contribution.

When signal strength quality is in fall due to neighbor's node remoteness, a discovery of road piece to rescue two more hops will be established that will be used in disconnection case.

To illustrate our idea, consider the following example (Figure 1). Nodes x1, x2 and xi are close two by two and neighbor to $\mathrm{xs}$ on one side and $\mathrm{xd}$ the other. $\mathrm{S}$ is the source and $\mathrm{D}$ is the destination.

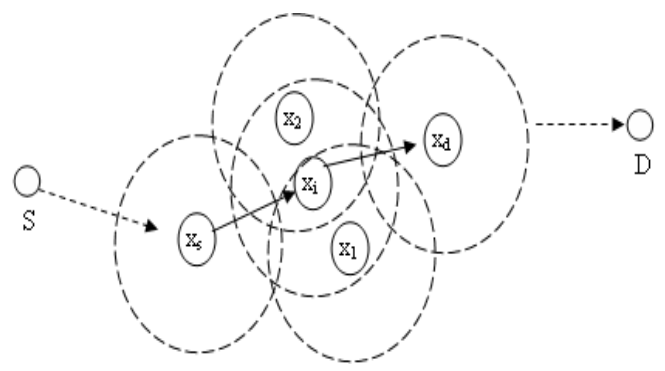

Fig.1. Vicinity nodes Graph

When node xi moves away due to mobility, its signal weakens (tends towards zero) and disconnection probability increases.
Two cases are to consider:

- $\mathrm{Xi}$ move towards $\mathrm{xd}$ and leave the range of $\mathrm{xs}$ (break of the segment [xs, xi]), then xs tries to rebuild road pieces $[\mathrm{xs}, \mathrm{xp}, \mathrm{xi}, \mathrm{xd}]$ (Figure 2) where $\mathrm{p}$ is one of neighbors at one hop from $\mathrm{xs}$ (in the example: $\mathrm{p}=1$ or 2 ; see Fig. 1 ).

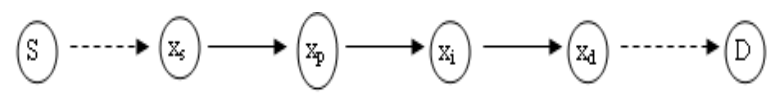

Fig.2. Rebuilding in xs Side

- Xi move towards $\mathrm{xs}$ and leave the range of $\mathrm{xd}$ (break of the segment [xi, xd]), then xs tries to rebuild road pieces [xs, xp, xd] (Figure 3 ) where $\mathrm{p}$ is one of the neighbors at one hop from xs (in the example: $\mathrm{p}=1$ or 2; see Fig.1) and from $\mathrm{xd}$.

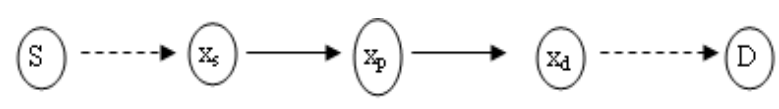

Fig.3. Rebuilding in xd Side

How these road segments are rebuilt (Figure 2 and 3), thing which is done of course in parallel with data transfer on active road is the idea implemented in PFAODV protocol in its phase of routing where metric named "signal strength" is used to determine if link is stable or not.

It is necessary noted that it is very difficult to predict exact probability that link will be broken in the near future but it is possible to estimate the relative stability of connection based on signal strength recent values received on this link.

Signal strength calculation obeys to one of these models: SSA (Signal Stability-based Adaptive Routing [9]), ABR (Associativity-Based Routing) or SBM (Advanced signal strength based link stability estimation model) [38].

\section{PF_Aodv Routing}

Routing in Ad hoc networks consist of two phases: discovery and maintenance road. The discovery phase is unchanged (without change) and is identical to original version AODV.

\section{C1. Route Maintenance:}

As in AODV, it is based on sending Hello message to neighboring initiated by nodes on active route at regular intervals and very short.

The hello packet is modified to contain information on vicinity signal strength i.e. Signal Strength Value (ValSS). According to Val-SS, Hello messages are two types: Ordinary (H_Ordinary) and Handoff (H_Handoff).

NB: if Signal Strength value is negative then the hello message is H_Handoff otherwise is H_Ordinary. It takes negative value to indicate to the node that we are rebuilding segment phase and not for maintenance.

H_Odinary message is initiated by nodes of active route to their neighbors, but the H_Handoff is initiated by a node said handoff which is selected by neighbors with 
most stable link and delegated for sending this message to his neighbors.

The signal strength is used to predict and identify all neighbor nodes and stored in new neighbors table "NGR_SIG" at each node compared to selected threshold value so that we can rebuild a road segment or backup or cache before active route fails.

NB: interval between two (02) consecutive H_Ordinary messages sent must be selected so that it allows the treatment of two (02) H_Handoff packets between edge ends to be reconstructed.

By receiving Hello packet, node decides to update the table NGR_SIG and inserts only the new neighbors with associated Val- SS or it updates it for former neighbors. For active route neighbors, signal strength value reached the threshold; a procedure denoted "Handoff» is launched to determine another segment which will be used in case of current path disconnection at any node.

Table structure is as follows:

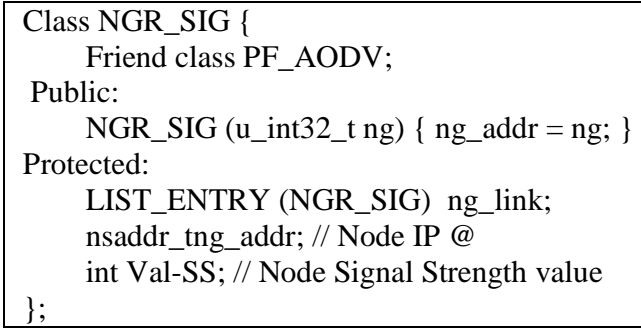

For NGR_SIG management, two functions (Insert and Delete) are implemented.

- NGR-Insert (node's neighbors, Val-SS): insert node neighbors on active route.

- NGR-Delete (node's neighbors): delete node neighbors on active route (delete NGR_SIG entry).

Among important fields to convey in H_Handoff packet are: @ IP node, IP @ next stable node, hop count to reach destination, sequence number and link Life time.

H_Handoff packet is sent between neighbors and each of its sides (edge ends) must especially modify its routing table for parameter Next Hop and Life time.

In general, each node must:

- Check signal strength value (Val_SS) for next and previous nodes that are part of active route for an interval time "th".

- Detect if link with the previous or next node of active road, its Val-SS is declining relative to "Threshold" value to launch "Handoff” procedure.

If node $\mathrm{xi}$ is close to $\mathrm{xs}$, xs initiate Handoff (probable that $[\mathrm{xd}$-xi] segment break)

For a predecessor (xp) of xi, finding the best neighbor $\mathrm{xh}$ such as $\mathrm{xh}$ is close to xi (i.e take only next neighbor of xi. Node $x h$ is considered as handoff node. If $x h$ has a road to $\mathrm{xd}$, the segment [xs---xh---xd] is rebuilt otherwise local repair as in AODV will be initiated.

If node $\mathrm{xi}$ is close to $\mathrm{xd}$, xs initiate Handoff (probable that [xs-xi] segment break), $\mathrm{xs}$ is considered as handoff node.

Evaluate_Link procedure is performed by each node of an active road when they receives H_Ordinary packet.

\section{C2. Algorithms}

Procedure Evaluate_Link

1. Compare "Val_SS" to "Threshold"
2. If it is greater than threshold, everything is good so
nothing to do and go to 4 (stop).
3. Otherwise launch procedure "Handoff" with
H_Handoff packet
4. End.

Procedure Handoff (packet: H_Handoff, node: xi, number of execution)

1. Find the best neighbor (xp) (greatest Val-SS of xi)

2. If it does not exist, a local repair as in the AODV will be initiated if failure occurs.

3. If it exists, $\mathrm{H}$ is handoff node, broadcasting $\mathrm{H} \_$Handoff packet between $\mathrm{P}$ and $\mathrm{H}$ and then modify Next hop from $\mathrm{P}$ to $\mathrm{D}$ by $\mathrm{H}$.

4. Verify if node $\mathrm{H}$ has a road to $\mathrm{D}$.

5. If it is the case, the segment is rebuilt and go to 7 (End)

6. Otherwise re-launch the "Handoff" procedure for node $\mathrm{H}$ (for only one execution).

7. End

NB: the number of execution is less or equal to 2 since we are interested in a reconstruction of two (02) segments at the maximum.

\section{SIMULATION}

Network Simulator (Ns2) [39] version 2.31 is used to investigate and analyze proposed idea. Simulation context consists of 20 nodes in a region $800 \times 600 \mathrm{~m} 2$. Random Way Point (RWP) mobility model is used and the transmission range is set to $250 \mathrm{~m}$ for ideal unobstructed. Nodes moves at average speed of $5 \mathrm{~m} / \mathrm{s}$. Two scripts are used, the first (cbrgen.tcl) for randomly generating traffic for Constant Bit Rate (CBR) of 512 bytes according to UDP protocol and the second (Setdest) to produce mobility scenarios. Simulation time is sets to $120 \mathrm{~s}$ for all tests [24].

\section{A. Constraints}

Mobility in Ad hoc network makes nodes out of reach of their neighbors and therefore links and paths which they are parts become invalid necessitating routes discovery process launch again

Nodes have limited energy, so it is imperative to best manage it as long as possible. Density (The average number of neighbors per node) and size (space) also have an impact on MANET performance [24].

\section{B. Metrics}

Evaluate network performances is to find if it is able to 
minimize packets loss, i.e. ensure if possible a transfer loss close to zero (quality criterion). Control load is required for network management but it consumes some bandwidth, over this rate is high, performance network degrades but conversely they are better. Taking into account characteristics (capacity) of physical links and current flow sharing them, when throughput is higher, bandwidth is used efficiently. For some applications, it is not enough to transmit large data quantity (high speed) without loss, but it is imperative to transmit it if possible faster, i.e. reduced delay in real-time applications [24].

\section{Curves \& Discussions}

The proposed solution is evaluated in different situations (mobility, density, etc.) according to the following parameters: data transfer rate or throughput in kbps, time taken between data packet transmission and reception (i.e. Average end- to-end delay (e2e)) shorter it is a desired goal. Packet Delivery ratio (PDR) describes the ratio of successfully delivered packets and packets lost ratio is the rapport among successfully received and sent packets. Network reliability is inversely proportional to this ratio. Network management is ensured through control packets estimated by Normalized Routing Load or Normalized Overhead Load (NRL or NOL) expressing overload network.

In the following, we study the effect of mobility (Respectively: Transmission rates) on parameters mentioned above by going from a stationary state (low mobility) (i.e. pause time greater or equal to 200s) to high mobility (i.e. pause time equal to zero) (Respectively: by varying transmission rates from $0.2 \mathrm{~Kb} / \mathrm{s}$ to $10 \mathrm{~Kb} / \mathrm{s}$ ) [24].

\section{C1. Discussions}

Figures 4 and 5 show that modified protocol (PF_AODV) improve throughput in case of low and medium mobility (Pause Time from 200s to 50s) and high transmission rate (between 1.5 and $10 \mathrm{~Kb} / \mathrm{s}$ ), whereas for strong mobility and low transmission rate (between 0 and $1.5 \mathrm{~Kb} / \mathrm{s}$ ) we notice that both protocols (AODV and PF_AODV) offer the same performances. This phenomenon can be explained by the impact of mobility on links stability where failure prediction in a stable and moderate environment is efficient. Note that throughput is proportional to the mobility and the transmission rate in both protocols.

The average end-to-end delay is in a gap of about 10s for transmission rate between 2 and $10 \mathrm{~Kb} / \mathrm{s}$ (Fig.7) in favor to PF_AODV protocol (over the AODV) but this gap is approximately of 5s (Fig.6) in the benefit of the version modified for a low and average mobility (up to a pause time of 20s), this is due to the availability of several stable links between neighboring nodes being a part of an established path. In the case of low transmissions rate $(<2 \mathrm{~Kb} / \mathrm{s})$ and a very strong mobility, the original version offers better results than the modified one which can be explained by the presence of an important overhead what slowed down considerably data transmissions and generate a consequent delay. Note that the average end-to-end delay is proportional to mobility and inversely proportional to the transmission rate for both protocols.

The prediction mechanism of routes rupture allowed the modified version (PF_AODV) to have better paths than basic version and consequently it minimizes largely packet loss in all situations and according constraints used in our simulation. (Fig. $8 \& 9$ shows respectively the cases of mobility and transmission rate). We noted that the transmission rate and the packet loss rate vary similarly; but according to mobility constraint nothing can be expected unless the proposed protocol gives a better result than the basic protocol

The PDR based on PF_AODV protocol shows acceptable values whether in mobility and transmission rate context (Fig.10, 11). NRL ratio is more or less balanced and ratio concerning both protocols is of bordering one value, i.e. more successful transfer induces more control packets (Fig.13). For measures based on the mobility constraint, this ratio is high in certain cases due to generation of more control packets to restore broken paths especially in AODV protocol (Fig.12). We can conclude that mobility and transmission rate have a direct impact on both parameters (PDR or NRL). The PDR is proportional to both parameters however NRL is inversely proportional to mobility and transmission rate.

\section{C2. Curves}

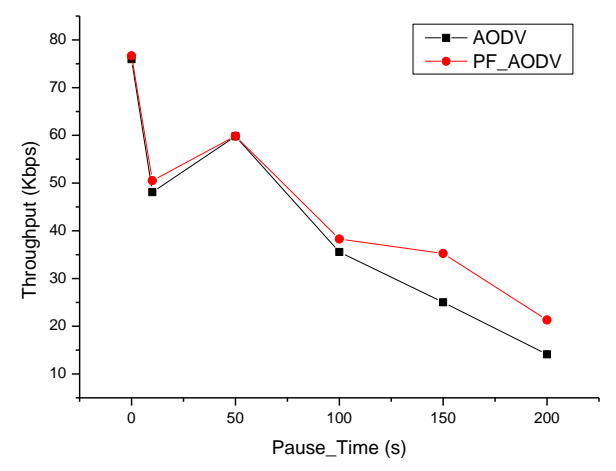

Fig.4. Throughput Vs Pause_Time

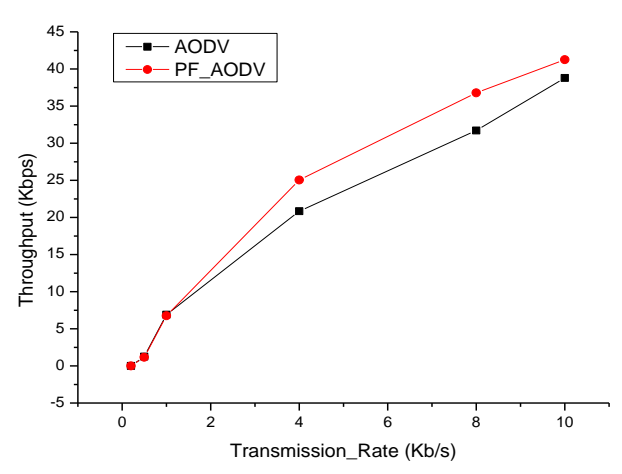

Fig.5. Throughput Vs Transmission_Rate 


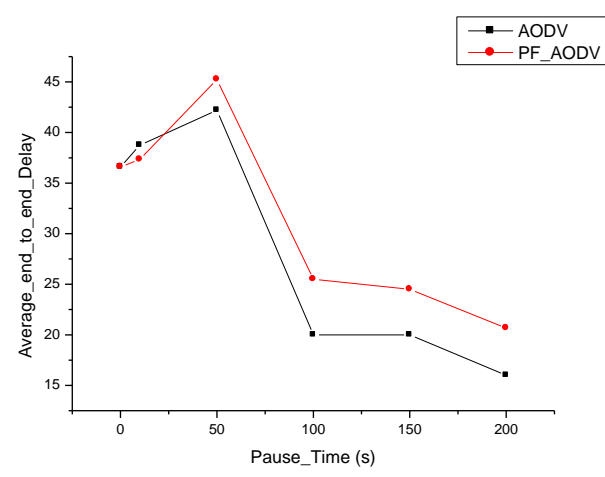

Fig.6. Average_end_to_end_Delay Vs Pause_Time

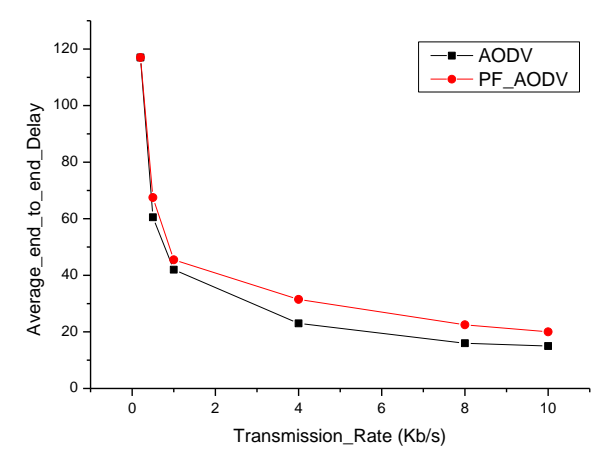

Fig.7. Average_end_to_end_Delay Vs Transmission_Rate

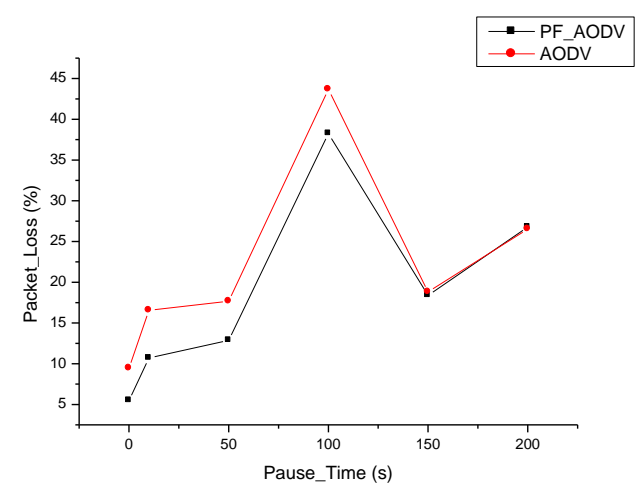

Fig.8. Packet_Loss_Ratio Vs Pause_Time

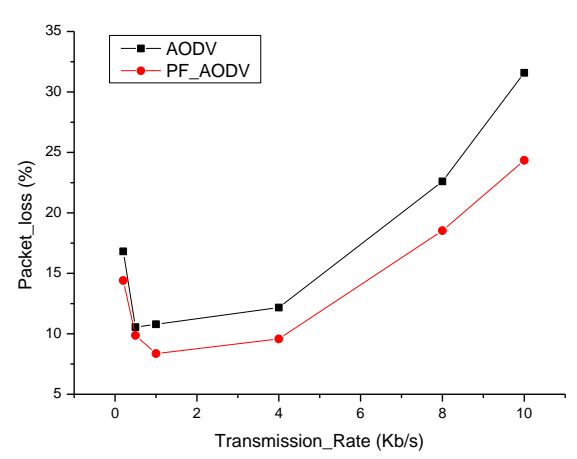

Fig.9. Packet_Loss_Ratio Vs Transmission_Rate

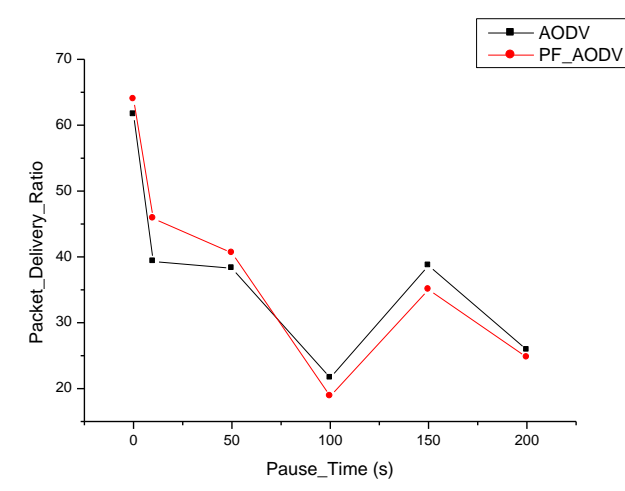

Fig.10. Packet_Delivery_Ratio Vs Pause_Time

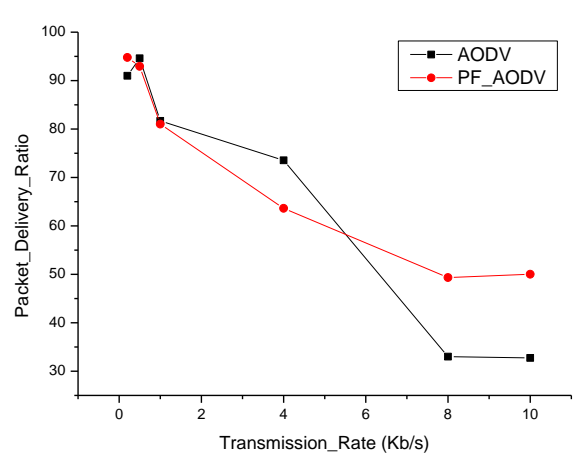

Fig.11. Packet_Delivery_Ratio Vs Transmission_Rate

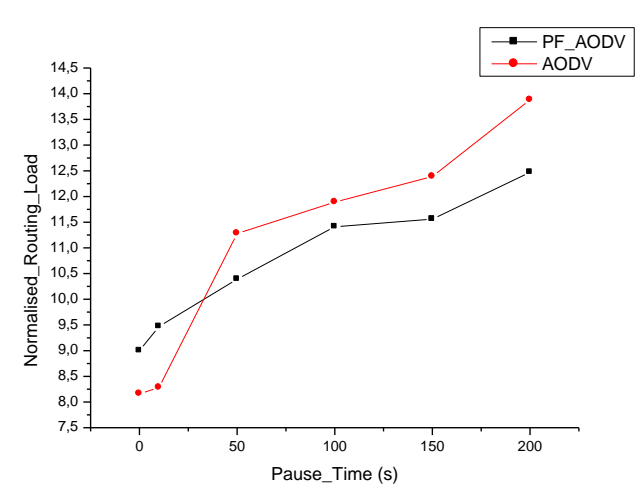

Fig.12. Normalised_Routing_Load Vs Pause_Time

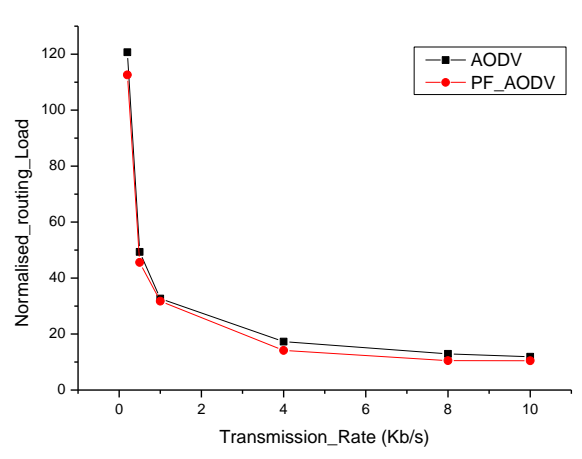

Fig.13. Normalised_Routing_Load Vs Transmission_Rate 


\section{CONCLUSION \& PERSPECTIVES}

In this paper, the proposed protocol (PF_AODV) based on AODV, has improved QoS for applications in MANETs certainly in context proposed in our simulation.

Using signal strength to predict future disconnection of road in use and to provide another segment (cache link) before break occurs will be greatly minimizes control traffic generated during roads reconstruction phase as it does in the original version.

The simulation results shows that PF_AODV provides better performance in terms of throughput, loss and delay. Future extensions of AODV protocol to add control admission to handle each traffic type separately and determine what kind of traffic should be penalized to free up bandwidth to support traffic priority.

Another direction in addition to the idea developed in this paper is to regulate conflicts caused by more than one procedure launch to ensure handoff phase between neighboring nodes to minimize control load is to introduce a random delay before each node initiates handoff phase, one node initiates this phase earlier than other or to promote nodes near the destination, if not; source recovery in AODV will better do.

\section{REFERENCES}

[1] E. Crawley, R. Nair, B. Rajagopalan and H. Sandick, «A Framework for QoS-based Routing in the Internet IETF RFC2386», 2002.

[2] «QoS Forum,» July 1999. [On line]. Available: http://www.qosforum.com.

[3] S. Chakrabarti and A. Mishra, «Quality of service challenges for wireless mobile Ad hoc networks,» Wiley J. Wireless Commun and Mobile Computing, vol. $4, \mathrm{n}^{\circ} 12$, p. 29-153, 2004.

[4] P. Periyasamy and E. Karthikeyan, «Survey of Current Multipath Routing Protocols for Mobile AD Hoc Networks,» International Journal of Computer Network and Information Security (IJCNIS), vol. 5.12, p. 68, 2013.

[5] C. Perkins, E. Belding-Royer and S. Das, «Ad hoc ondemand distance vector routing,» RFC 3561, IETF, 2003.

[6] N.-C. WANG and C.-Y. LEE, «A reliable QoS aware routing protocol with slot assignment for mobile ad hoc networks,» Journal of Network and Computer Applications, vol. 32, n¹6, pp. 1153-1166, 2009.

[7] H.-J. Cha, I.-S. Han et H.-B. Ryou, «QoS routing mechanism using mobility prediction of node in ad-hoc network», In Proceedings of the 6th ACM international symposium on Mobility management and wireless access. ACM, 2008.

[8] J. LIU and F.-M. LI, «An Improvement of AODV Protocol Based on Reliable Delivery in Mobile Ad hoc Networksll,» In Fifth International Conference on Information Assurance and Security, 2009.

[9] R. Dube, C. D. Rais, K.-Y. Wang and S. Tripathi, «Signal Stability-Based Adaptive Routing (SSA) for Ad Hoc Mobile Networks,» IEEE Personal Communications, 1997.

[10] S. M. Abdule et S. Hassan, «Divert Failure Route Protocol Based on AODV» In Network Applications Protocols and Services (NETAPPS), 2010 Second International Conference on. IEEE, 2010.

[11] Y. Hwang et P. Varshney, «An adaptive QoS routing protocol with dispersity for ad-hoc networks,» chez System Sciences, 2003. Proceedings of the 36th Annual Hawaii International Conference on. IEEE, 2003.

[12] R.-S. Chang and S.-J. Leu, «Long-lived path routing with received signal strength for ad hoc networks» In Wireless Pervasive Computing, 1st International Symposium on. IEEE, 2006.

[13] N. Sarma and S. Nandi, «Route stability based QoS routing in mobile Ad Hoc networks,» Wireless Personal Communications, vol. 54, ${ }^{\circ}$ 11, pp. 203-224, 2010.

[14] J. N. Boshoff and A. S. J. Helberg, «Improving QoS for real-time multimedia traffic in ad-hoc networks with delay aware multi-path routing» In Wireless Telecommunications Symposium, 2008. WTS 2008. IEEE., 2008.

[15] R. Ghanbarzadeh and M. R. Meybodi, «Reducing message overhead of AODV routing protocol in urban area by using link availability prediction,» In Computer Research and Development, 2010 Second International Conference on. IEEE, 2010.

[16] M. Zarei, K. Faez and J. M. d Nya, «Modified Reverse AODV routing algorithm using route stability in mobile ad hoc networks,» In Multitopic Conference, 2008. INMIC 2008. IEEE International. IEEE, 2008.

[17] S. J. Philip and V. Anand, «Mobility aware path maintenance in ad hoc networks,» In Proceedings of the 2009 ACM symposium on Applied Computing. ACM, 2009.

[18] M. Maleki, K. Dantu and M. Pedram, «Lifetime prediction routing in mobile ad hoc networks,» Wireless Communications and Networking 2003, WCNC 2003. 2003 IEEE, vol. 2, 2003.

[19] M. Veerayya, V. Sharma and A. Karandikar, «SQ-AODV: A novel energy-aware stability-based routing protocol for enhanced QoS in wireless ad-hoc networks,» In Military Communications Conference. MILCOM 2008. IEEE, 2008.

[20] Q. Li, C. Liu and H.-h. Jiang, «The routing protocol of AODV based on link failure prediction,» In Signal Processing, 2008. ICSP 2008. 9th International Conference on. IEEE, 2008.

[21] A. T. Haghighat and O. J. Khoshrodi, «Stable QoS routing by AODV protocol,» In Software, Telecommunications and Computer Networks,2007. SoftCOM2007. 15th International Conference on. IEEE, 2007.

[22] D. Satyanarayana and S. V. Rao, «Link failure prediction QoS routing protocol for MANET,»ICTES 2007, p. 1031 - 1036, 2007.

[23] J. Novatnack, L. Greenwald and H. Arora, «Evaluating Ad hoc Routing Protocols with Respect to Quality of Service. Technical Report DU-CS-04-05» Department of Computer Science, Drexel University, Philadelphia, PA, 2004.

[24] M. Sedrati, A. Bilami and M. Benmohamed, «M-AODV: AODV variant to improve quality of service in MANETs,» arXiv preprint arXiv, p. 1104.1186, 2011.

[25] R. Braden, L. Zhang et al, «Integrated Services in the Internet Architecture: an Overview,» RFC 1633, 1994.

[26] S. Blake, D. Black, M. Carlson, E. Davies, Z. Wang et W. Weiss, Architecture for Differentiated Services, RFC 2475, 1998. H. Xiao, W. Seah,

[27] H. Xiao, W. Seah, A. Lo and K. Chu, «A Flexible Quality of Service Model for Mobile Ad-Hoc Networks,» the Proceedings of The IEEE 51st Vehicular Technology, pp. 445-449, 2000.

[28] A. Gahng-Seop, A. Campbell, A. Veres and L.-H. Sun, «SWAN: Service Différentiation in Wireless Ad Hoc Networks.,» IEEE Transactions On Mobile Computing, 
vol. 1, n 13, 2002.

[29] IMAQ, «An integrated mobile ad hoc QoS framework,» 2006. [On line]. Available: http://cairo.cs.uiuc.edu/adhoc.

[30] A. Orda and A. Sprintson, «Precomputation Schemes for QoS Routing., vol. 11, 578-591.,» IEEE/ACM Transaction on Networking, vol. 11, pp. 578-591, 2003.

[31] R. Sharma and J. Malhotra, «Sharma, Richa; Malhotra, Jyoteesh "Performance Evaluation of AODV and GOD for Qos Aware Applications through Realistic Conditions in VANET,» International Journal of Computer Network and Information Security (IJCNIS) , vol. 7.11, p. 64, 2015.

[32] T. Bhatia and A. K. Verma «QoS Comparison of MANET Routing Protocols» I. J of Computer Network and Information Security (IJCNIS), vol. 7.9, p. 64, 2015.

[33] L. Xiao, J. Wang and K. Nahrstedt, «The Enhanced Ticket-based Routing Algorithm.,»In Proc. of IEEE ICC 2002, New York, NY, 2002.

[34] P. Sinha, R. Sivakumar and V. Bharghanan, «CEDAR: a Core-Extraction Distributed Ad-Hoc Routing Algorithm,» IEEE Journal on Selected Areas in Communications, vol. 17, n 18, 1999.

[35] G. S. Ahn, A. T. Campbell, S. B. Lee and X. Zhang, «INSIGNIA» IETF MANET Internet Draft draft-ietfmanet-insignia-01.txt, 1999.

[36] C. Boulkamh, A. Bilami, A. Saib and M. Sedrati, «AODV_MC: Un Protocole de Routage AODV avec Minimum de Contrôle de Routage», Jeesi09, Alger, 2009.

[37] M. Tahar Abbes, M. Senouci and B. Kechar, «Impact of Model Mobility in Ad Hoc Routing Protocols,» International Journal of Computer Network and Information Security (IJCNIS), vol. 4.10, p. 47, 2012.

[38] C. YAWUT, «Adaptation à la mobilité dans les réseaux Ad hoc,» Thèse de Doctorat, Université de Toulouse, 2009.

[39] K. Fall and K. Varadhan «The NS Manual» Vint Project,
UC Berkeley, DARPA, USC/ISI, and Xerox PARC, 2002.

\section{Authors' Profiles}

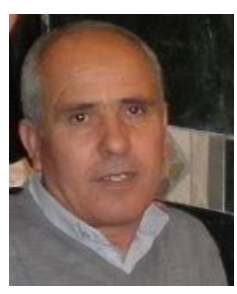

Sedrati Maamar received his Engineering degree in 1985 from UMC Constantine, Algeria and he obtained the Ph.D. degree in 2011 from UHL Batna University, Algeria. $\mathrm{He}$ is currently serving as assistant professor and member of LaSTIC laboratory at the Computer Science Department, University of Batna2, Algeria. His research interests include computer networks, Internet technologies and mobile computing, security, quality of service for Multimedia applications in wireless and mobile networks and several aspects of the Internet of Things (IoT). He serves as a technical program committee in many national and international Algerian conferences such as ICACIS, CN2TI and ICCSA.

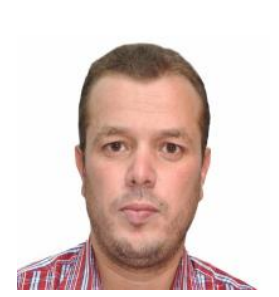

Benyahia Abderezzak received his Engineering degree in 2008 from UHL Batna, Algeria. He is currently Ph.D. student, member of LaSTIC laboratory and Assistant Professor in the Computer Science Department of Batna2 University. Research subjects include Information Systems, Computer networks, Mobile Wireless ad hoc networks (MANets), Wireless Sensor Networks, Modeling and simulation.

How to cite this paper: Sedrati Maamar, Benyahia Abderezzak,"Predict Link Failure in AODV Protocol to provide Quality of Service in MANET", International Journal of Computer Network and Information Security(IJCNIS), Vol.8, No.3, pp.1-9, 2016.DOI: 10.5815/ijcnis.2016.03.01 